\title{
Immunocytochemical Localization of Noncollagenous Proteins in Mineralized
}

\section{Tissues}

\author{
Ling Chen, Robin Jacquet, Elizabeth Lowder and William J. Landis
}

Department of Polymer Science, University of Akron, Akron, Ohio, USA

Normally mineralized vertebrate tissues such as bone, tooth dentin, and calcified tendons are biological composite materials in which apatite and collagen are their respective major inorganic and organic components. Presently there remain many questions about mineral formation and its mechanism(s) in these tissues. Collagen fibrils are considered to serve as a template for the nucleation and growth of apatite [1]. Other studies suggest that certain charged noncollagenous proteins (NCPs) also play an essential role in mediating the mineralization process and influencing both the nucleation and growth of apatite [2]. However, while extensive information is known about collagen, relatively less is understood about the NCPs, particularly bone sialoprotein (BSP), osteopontin (OPN), osteocalcin (OC) and others implicated in mineralization events. Calcified avian tendons have been used as a model for studying vertebrate mineralization because of their apparently simpler structure compared to their complex counterparts such as bone and dentin. Work with turkey tendons has demonstrated that their progressive deposition of apatite provides valuable information about spatial and temporal events of mineralization $[3,4]$. The study here attempts to characterize more completely the presence, location, and possible interaction of NCPs with collagen and extracellular matrix in the avian calcified tendon model. The principal method of immunocytochemistry is used to gain insight into possible roles of NCPs in the mineralization process of vertebrates.

Gastrocnemius tendons were dissected from legs of freshly sacrificed 12-15-week-old male turkeys. Tissues were immediately pinned on paraffin in a container and immersed in 4\% formaldehyde/1-2.5\% glutaraldehyde in $0.1 \mathrm{M}$ cacodylate buffer ( $\mathrm{pH}$ 7.4). Specimens were examined by X-ray radiography and then intermediate regions between identified heavily calcified and uncalcified tendon zones were dissected, processed and embedded in LR White or epoxy resin. Thin sections $(\sim 80 \mathrm{~nm})$ were cut and collected on carbon/Parlodion-coated Ni grids. Immunocytochemistry followed a previous protocol [2]. Briefly, sections on grids were incubated with phosphate buffered saline (PBS) for 5 min. An additional on-grid decalcification process using EDTA was applied to some samples to retrieve antigens, especially osteocalcin (OC). Grids were treated with blocking agent containing 1\% bovine serum albumin (BSA) for $10 \mathrm{~min}$ and then incubated with primary polyclonal antibody solution at $4^{\circ} \mathrm{C}$ overnight. After washing three times with PBS, grids were treated with 1\% BSA for $5 \mathrm{~min}$ followed by a $6 \mathrm{~nm}$ protein Agold conjugate solution for $1 \mathrm{hr}$ at ambient temperature. Sections on grids were next washed three times with PBS, rinsed with Milli-Q water, air-dried overnight, and finally examined by transmission electron microscopy (TEM). No other counterstaining treatments were used unless described specifically.

X-ray imaging revealed radiopaque regions corresponding to mineralized tendon zones (Figure 1). NCP immunolocalization (Figure 2) demonstrated BSP associated with small apatite platelets in extrafibrillar collagen regions of the tendon. Intrafibrillar regions did not show BSP labeling. Relationships between OPN and tissue were not clear because of the sparsely distributed gold nanoparticles over tendon sections. Different from the observations in chicken bones [2], the location and amount of labeled OPN could not be correlated conclusively with the mineralization process in turkey tendons [5]. As apatite may mask epitopes accessible to the antibodies used in this study, EDTA decalcification was used as a 
possible means to help retrieve antigens. After EDTA treatment, abundant OC was found in mineralized zones of intrafibrillar collagen regions. Labeling of OC was almost absent without decalcification (data not shown), a result consistent with immunohistochemistry reported by Kacena et al [6].

In conclusion, BSP appears to co-localize with small apatite crystals in the extrafibrillar regions of collagen in tendon. In tendon collagen intrafibrillar regions, abundant OC is identified after on-grid decalcification to unmask its epitopes. OPN is dispersed sparsely in tendon, and its localization in this manner precludes its correlation with apatite crystals. Associations between these NCPs, collagen and mineral in the tendon model provide further insight into vertebrate mineralization events [7].

\section{References:}

[1] FH Silver and WJ Landis, Connect Tissue Research 52 (2011), p. 242.

[2] MD McKee et al, Anatomical Record 228 (1990), p. 77.

[3] WJ Landis et al, Journal of Structural Biology 110 (1993), p. 39.

[4] WJ Landis, Journal of Ultrastructure and Molecular Structure Research 94 (1986), p. 217.

[5] WJ Landis et al, Bone and Mineral 17 (1992), p. 237.

[6] MA Kacena et al, Journal of Histotechnology 26 (2003), p. 105.

[7] The authors thank Drs. Donald Ott, Yan Cao, and Bojie Wang for help with TEM experiments at the University of Akron and the Department of Radiology staff for assistance with X-ray radiography at the Akron General Medical Center. Valuable discussions and gifts of antibodies from Drs. Marc McKee at McGill University and Louis Gerstenfeld at Boston University are greatly appreciated.

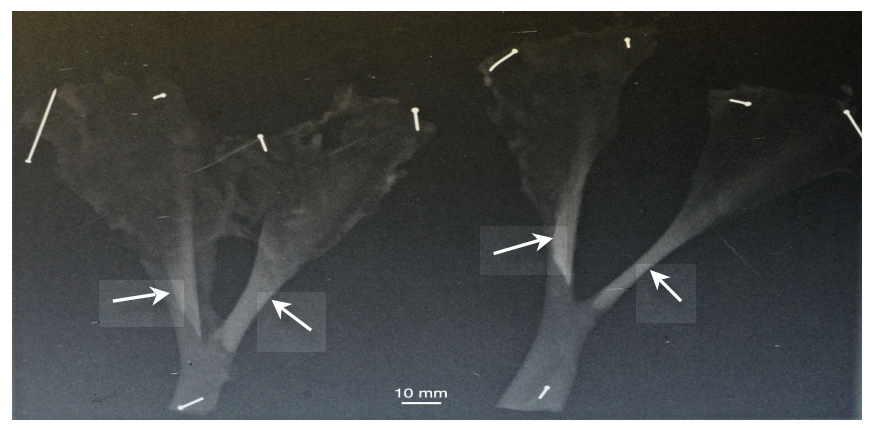

Figure 1. X-ray image of calcified turkey gastrocnemius tendons pinned to a paraffin wax slab. The radiopaque regions indicated by arrows are the mineralized tendon zones. Scale bar: $10 \mathrm{~mm}$
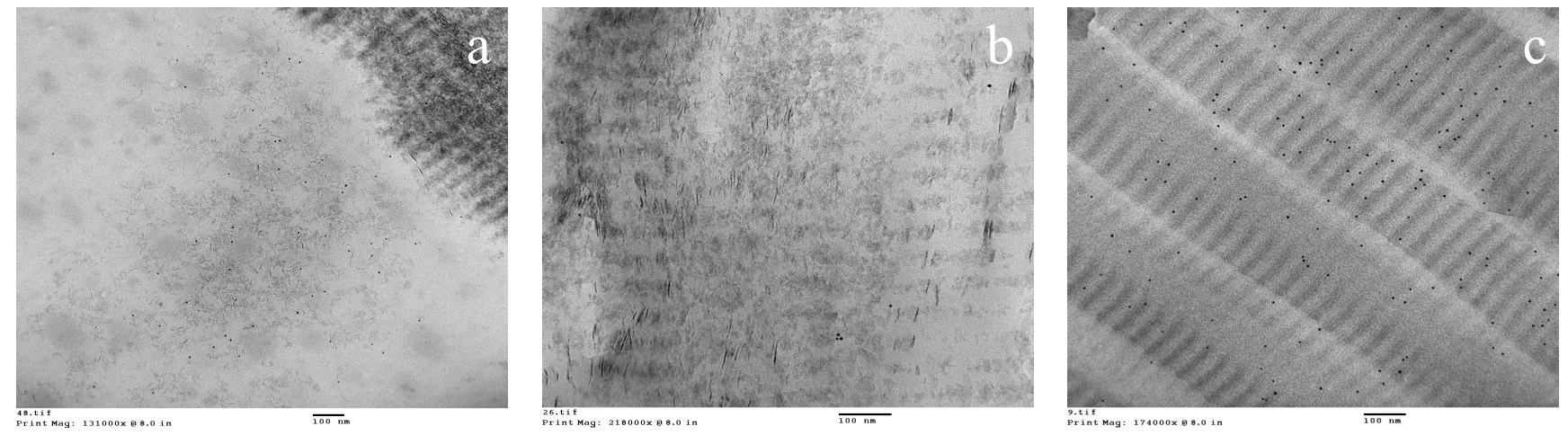

Figure 2. Immunogold-labeled NCPs: a) BSP; b) OPN; c) OC (after decalcification with EDTA and staining with $1 \%$ uranyl acetate). Scale bar: $100 \mathrm{~nm}$. 